GA II cannot distinguish the parents from normal individuals although the method measures half-normal activities in GA I parents (see Table 7). An explanation for this discrepancy could be that GDH is the limiting factor in this assay as indicated by the intermediate values in the obligate GA I heterozygotes. Halfnormal concentration of ETF or ETF-DH would then still be enough for full GDH activity. Another possibility is that the synthesis of the missing enzyme in GA II (i.e. ETF or ETF-DH) is regulated so the one gene that is functioning in GA II heterozygotes keeps working until a normal enzyme level is reached. This cannot occur in GA II homozygotes where both genes are defective.

Although the normal GDH activity measured with intact ETC as EA is a disadvantage in the diagnosis of GA II heterozygotes, it makes prenatal diagnosis of GA II homozygotes easier because of the high difference in enzyme activities between GA II heterozygotes and homozygotes.

\section{REFERENCES AND NOTES}

1. Christensen E, Brandt NJ 1978 Studies on glutaryl-CoA dehydrogenase in leucocytes, fibroblasts and amniotic fluid cells. The normal enzyme and the mutant form in patients with glutaric aciduria. Clin Chim Acta 88:267

2. Christensen $E 1983$ Improved assay of glutaryl-CoA dehydrogenase in cultured cells and liver: application to glutaric aciduria type I. Clin Chim Acta 129:91

3. Coude FX, Ogier H, Charpentier C, Thomassin G, Checoury A, AmedeeManesme O, Saudubray JM, Frezal J 1981 Neonatal glutaric aciduria type II: an X-linked recessive inherited disorder. Hum Genet 59:263

4. Dancis J, Hutzler J, Cox RP 1979 Familial hyperlysinemia: enzyme studies, diagnostic methods, comments on terminology. Am J Hum Genet 31:290

5. Deluca C. Kaplan NO 1958 Flavin adenine dinucleotide synthesis in animal tissues. Biochim Biophys Acta 30:6

6. Fazekas AG, Sandor T 1973 Studies on the biosynthesis of flavin nucleotides from $2-{ }^{14} \mathrm{C}$-riboflavin by rat liver and kidney. Can J Biochem 51:772

7. Goodman SI, McCabe ERB, Fennessey PV, Mace JW 1980 Multiple acyl-CoA dehydrogenase deficiency (glutaric aciduria type II) with transient hypersarcosinemia and sarcosinuria: possible inherited deficiency of an electron cosinemia and sarcosinuria: possible int
transfer flavoprotein. Pediatr Res 14:12

8. Gregersen N, Kølvraa S, Rasmussen K, Christensen E, Brandt NJ, Ebbesen F, Hansen FH 1980 Biochemical studies in a patient with defects in the metabolism of acyl-CoA and sarcosine: another possible case of glutaric aciduria type II. J Inher Metab Dis 3:67

9. Kølvraa S, Gregersen N, Christensen E, Hobolth N 1982 In vitro fibroblasts studies in a patient with $\mathrm{C}_{6}-\mathrm{C}_{10}$-dicarboxylic aciduria: evidence for a defect in general Acyl-CoA dehydrogenase. Clin Chim Acta 126:53

10. Lowry $\mathrm{OH}$, Rosebrough NJ, Farr AL, Randall RJ 1951 Protein measurement with the Folin phenol reagent. J Biol Chem 193:265

11. Przyrembel H, Wendel U, Becker K, Bremer HJ, Bruinvis L, Ketting D, Wadman SK 1976 Glutaric aciduria type II: report on a previously undescribed metabolic disorder. Clin Chim Acta 66:227

12. Rhead W. Mantagos S, Tanaka K 1980 Glutaric aciduria type Il: in vitro studies on substrate oxidation, acyl-CoA dehydrogenases and electrontransferring flavoprotein in cultured skin fibroblasts. Pediatr Res 14:1339

13. Saudubray JM, Coude FX, Demaugre F, Johnson C, Gibson KM, Nyhan WL 1982 Oxidation of fatty acids in cultured fibroblasts: a model system for the detection and study of defects in oxidation. Pediatr Res 16:877

14. Sweetman L. Nyhan WL. Trauner DA, Merritt TA, Singh M 1980 Glutaric aciduria type II. J Pediatr 96:1020

15. This research was supported by the Danish Medical Research Council.

16. The authors acknowledge the expert technical assistance of Mrs. Eva Korup, Mrs. Vibeke Winter, and Mrs. Mette Houman Møller.

17. Requests for reprints should be addressed to: Ernst Christensen, Department of Clinical Genetics. University of Copenhagen, Rigshospitalet 4062, Blegdamsvej 9, 2100 Copenhagen, Denmark.

18. Received for publication August 6,1983

19. Accepted for publication October 19, 1983

\title{
Phototherapy for Neonatal Jaundice: in Vitro Comparison of Light Sources
}

\author{
JOHN F. ENNEVER, ${ }^{(20)}$ MARK SOBEL, ANTONY F. MCDONAGH, AND WILLIAM T. SPECK \\ Department of Pediatrics, Rainbow Babies and Childrens Hospital, Case Western Reserve University School of \\ Medicine, Cleveland, Ohio USA [J.F.E., M.S., W.T.S.]; and Department of Medicine and Liver Center, \\ University of California, San Francisco, California USA [A.F.M.]
}

\section{Summary}

Phototherapy results in the conversion of bilirubin to more water-soluble isomers. Six clinically used phototherapy lamps which differ in their emission spectra have been compared in their ability to produce configurational and structural isomers of bilirubin in vitro. For all of the lamps, the initial rate of configurational isomerization was highly correlated $(r=0.969)$ with the intensity of irradiation falling within the bilirubin absorption band. The percentage of the total bilirubin converted to the configurational isomer at equilibrium was dependent upon the spectral distribution of the lamp, and was greatest $(26.2 \pm 1.3 \%)$ with the special blue lamp, which has a narrow spectral output centered at $445 \mathrm{~nm}$. The rate of formation of the structural isomer, lumirubin, was generally dependent upon the intensity of irradiation within the bilirubin absorption band.

\section{Abbreviation}

HEPES, $N$-2-Hydroxyethylpiperazine- $N$ '-2-ethanesulfonic acid
Despite the widespread use of phototherapy for the prevention and treatment of neonatal jaundice, debate continues over the most effective light source $(2,6,15-19)$. Thus, several phototherapy units which differ in spectral distribution and intensity have been recommended. These recommendations are based on empirical studies which compared the effect of different light sources on serum bilirubin concentration in jaundiced infants $(15-19)$ or congenitally jaundiced Gunn rats (2). A limitation of such studies is that the measured effect of phototherapy, i.e. decline in serum bilirubin, is a secondary and delayed response $(3,10)$ (Ennever, Knox, Denne, and Speck, submitted). The primary effect of the treatment is rapid conversion of bilirubin to excretable photoproducts $(8,13,14)$ (Ennever et al., submitted). These include yellow compounds which are isomers of bilirubin (i.e. which have the same chemical formula) $(11,13)$. The decline in serum bilirubin requires transport of these photoisomers from their site of formation to the liver where they are excreted in the bile (14). Recent studies have shown that the rates of excretion of these photoproducts are different in the 
human newborn infant than in the Gunn rat (14) (Ennever et al., submitted). Therefore, data derived from the Gunn rat on the relative efficacy of various phototherapy lamps may not be directly applicable to the human neonate.

Previous studies have shown that, during phototherapy of jaundiced infants or irradiation of bilirubin-human albumin solutions in vitro, the most rapid photochemical reaction is the formation of a configurational isomer of bilirubin, designated as $4 Z, 15 E$-bilirubin $(8,12)$. When the reaction is carried out in vitro, an equilibrium is eventually established between the natural $4 Z, 15 Z$ form and the $4 Z, 15 E$ isomer $(6,12)$. An additional photochemical reaction, which occurs at a slower rate both in vitro and in vivo, is the formation of a structural isomer, designated lumirubin (11) (Ennever et al., submitted). Recently, we have shown that both the rate of formation (4) and the equilibrium concentration (6) of the $4 Z, 15 E$ configurational isomer produced by monochromatic irradiation in vitro is highly wavelength dependent. It is difficult to extrapolate these results to the broad spectrum lights commonly used in phototherapy. Therefore, we have selected several phototherapy lamps that differ in their spectral emission characteristics and have compared their ability to produce configurational and structural isomers of bilirubin in vitro. These data provide the background necessary for similar comparative studies in jaundiced infants.

\section{MATERIALS AND METHODS}

Bilirubin (Sigma Chemical Co., St Louis, MO), purified as previously described (9), was dissolved in $0.01 \mathrm{M} \mathrm{NaOH}$ and added to a solution of human serum albumin (fatty acid free, Sigma) in $0.15 \mathrm{M} \mathrm{NaCl} 0.05 \mathrm{~m}$ HEPES, pH 7.5. After addition of the alkaline bilirubin solution, the $\mathrm{pH}$ of the solution was restored to 7.4 by the addition of $0.01 \mathrm{M} \mathrm{HCl}$. Final concentrations were $44 \mu \mathrm{M}$ bilirubin and $88 \mu \mathrm{M}$ albumin.

Five of the light sources were fluorescent lamps (20 watt): special blue (Westinghouse F20T12/BB), blue (Sylvania F20T12/B), daylight (Sylvania F20T12/DA), green (Sylvania F20T12/G), and Vita-Lite (DuroTest). Before use, the lamps were aged for $100 \mathrm{~h}$ to obtain a stable spectral output (7). The one additional light source was a Cavitron PT 1400 phototherapy unit containing a tungsten-halogen filament lamp (General Electric type ENH)

Fifteen-milliliter samples of the bilirubin-albumin solution were placed in $30-\mathrm{ml}$ plastic culture flasks (Costar, Cambridge, $\mathrm{MA}$ ), resulting in a $3.5 \times 3.5 \times 1.3 \mathrm{~cm}$ volume of liquid. Samples were irradiated normal to the square surface, positioned $50 \mathrm{~cm}$ from either a bank of eight fluorescent lamps in a standard phototherapy canopy or from a single tungsten-halogen lamp. During irradiation, the samples were magnetically stirred and thermostated to $20^{\circ} \mathrm{C}$. Aliquots were removed at timed intervals and the formation of bilirubin photoproducts was followed by high pressure liquid chromatography on a $0.46 \times 25 \mathrm{~cm}$ ZorbaxODS column (Dupont Co., Wilmington DE) fitted with a precolumn. The eluant was $0.1 \mathrm{M}$ di-n-octylamine acetate in methanol, pH 7.7 (12). Chromatography was performed on a Varian model 5060 liquid chromatograph equipped with a model UV100 detector (Varian Associates, Walnut Creek, CA) set at 465 $\mathrm{nm}$ and a Hewlett-Packard (Avondale, PA) model 3390A reporting integrator. The integrated peak areas were not corrected for differences in the $465-\mathrm{nm}$ extinction coefficient among the various bilirubin isomers.

Emission spectra of the lamps were recorded on Cary model 118 or 210 spectrophotometers (Varian Associates). Emission spectra were recorded in the single beam mode with the phototherapy lamps substituted for the spectrophotometer light source. Irradiance at $50 \mathrm{~cm}$ was measured normal to each lamp with an International Light model IL700 radiometer and a SEE 400 broad-band detector fitted with a filter (type TBLU, International Light) which transmitted light between 350 and $530 \mathrm{~nm}$. The peak response of this detector-filter combination was at $468 \mathrm{~nm}$ with half-maximum points at 397 and $483 \mathrm{~nm}$.

\section{RESULTS AND DISCUSSION}

When solutions of bilirubin bound to human serum albumin are exposed to light, the fastest reaction is configurational isomerization about the meso double bond between carbons 15 and 16 [configurational isomerization at the C-4-C-5 meso double bond does not occur when bilirubin is bound to human albumin (11)]. This reaction can be represented by:

$$
4 Z, 15 Z \text {-bilirubin } \stackrel{h v}{\longrightarrow} 4 Z, 15 \text { E-bilirubin }
$$

where $h \nu$ represents a photon of frequency $\nu$. The results in Table 1 demonstrate that the initial rate of this reaction, expressed as the inverse of the time required to convert $10 \%$ of the native bilirubin to the $4 Z, 15 E$ isomer $\left(1 / T_{10}\right)$, is dependent upon the intensity of irradiation falling within the absorption band of bilirubin $\left(I_{400-500}\right)$. The correlation between these two parameters is 0.969 .

The configurational isomerization reaction (Equation 1 ) is photochemically reversible $(6,12)$ and must compete with the corresponding reverse reaction (the $4 E, 15 E$ isomer is not formed in detectable amounts):

$$
4 Z, 15 Z \text {-bilirubin } \underset{h \nu}{\stackrel{h \nu}{\rightleftarrows}} 4 Z, 15 \text { E-bilirubin }
$$

Thus, in a closed system, an equilibrium is eventually established. Because the two isomeric forms have overlapping, but not identical, absorption spectra (6), the position of this equilibrium (Equation 2) will depend upon the spectral distribution of the light source being used. With monochromatic irradiation, the percentage of the total bilirubin present as the $4 Z, 15 E$ isomer at equilibrium ranged from $16 \%$ with $490 \mathrm{~nm}$ light to greater than $40 \%$ with $390 \mathrm{~nm}$ light (6). The table gives the percentage of the total bilirubin present as the $4 Z, 15 E$ isomer at equilibrium with the six phototherapy lamps. The greatest equilibrium concentration of $4 Z, 15 E$ configurational isomer was achieved with the special blue lamp, which has a narrow spectral output centered at $445 \mathrm{~nm}$ (Fig. 1). Lower equilibrium concentrations were produced by the three fluorescent lamps with broad emission spectra [daylight, Vita-Lite, and blue (Fig. 1)]. A somewhat lower equilibrium concentration was produced by the tungsten-halogen lamp despite its rapid initial rate of isomerization. The least conversion of native bilirubin to the $4 Z, 15 E$ isomer was achieved with the green lamp [recently used experimentally in the treatment of neonatal hyperbilirubinemia (18)], which has little emission falling within the absorption band of bilirubin (Fig. 1).

Table 1 shows the percentage of the total bilirubin present as the structural isomer lumirubin after 20 and 60 min of irradiation with the six phototherapy lamps. The greatest conversion of bilirubin to lumirubin was achieved by the two lamps (special blue and tungsten-halogen) with the greatest irradiance within the bilirubin absorption band. The green lamp, with the least emission in the 400 to $500 \mathrm{~nm}$ region, produced lumirubin at a rate comparable to that of the remaining three fluorescent lamps (daylight, Vita-Lite, and blue). This result is somewhat surprising. Some enhancement of the rate of lumirubin formation by the green lamp would be expected because this lamp forms the least $4 Z, 15 E$ isomer. The $4 Z, 15 E$ isomer is not a precursor of lumirubin (11) and its presence diminishes the rate of lumirubin formation both by reducing the concentration of native bilirubin and by acting as an internal filter. An additional enhancement of the rate of lumirubin formation by the green lamp would result if this photochemical reaction is favored by light at longer wavelengths. The action spectrum for conversion of bilirubin to lumirubin has not been measured and will be difficult to determine because of the competing and much more rapid formation of $4 Z, 15 E$-bilirubin. Recent studies indicate that lumirubin is formed directly from bilirubin and not from the $4 Z, 15 E$ isomer (11). Therefore, the action spectrum for lumirubin formation in vitro is probably coincident with, or at least contained within, the absorption spectrum of bilirubin. 


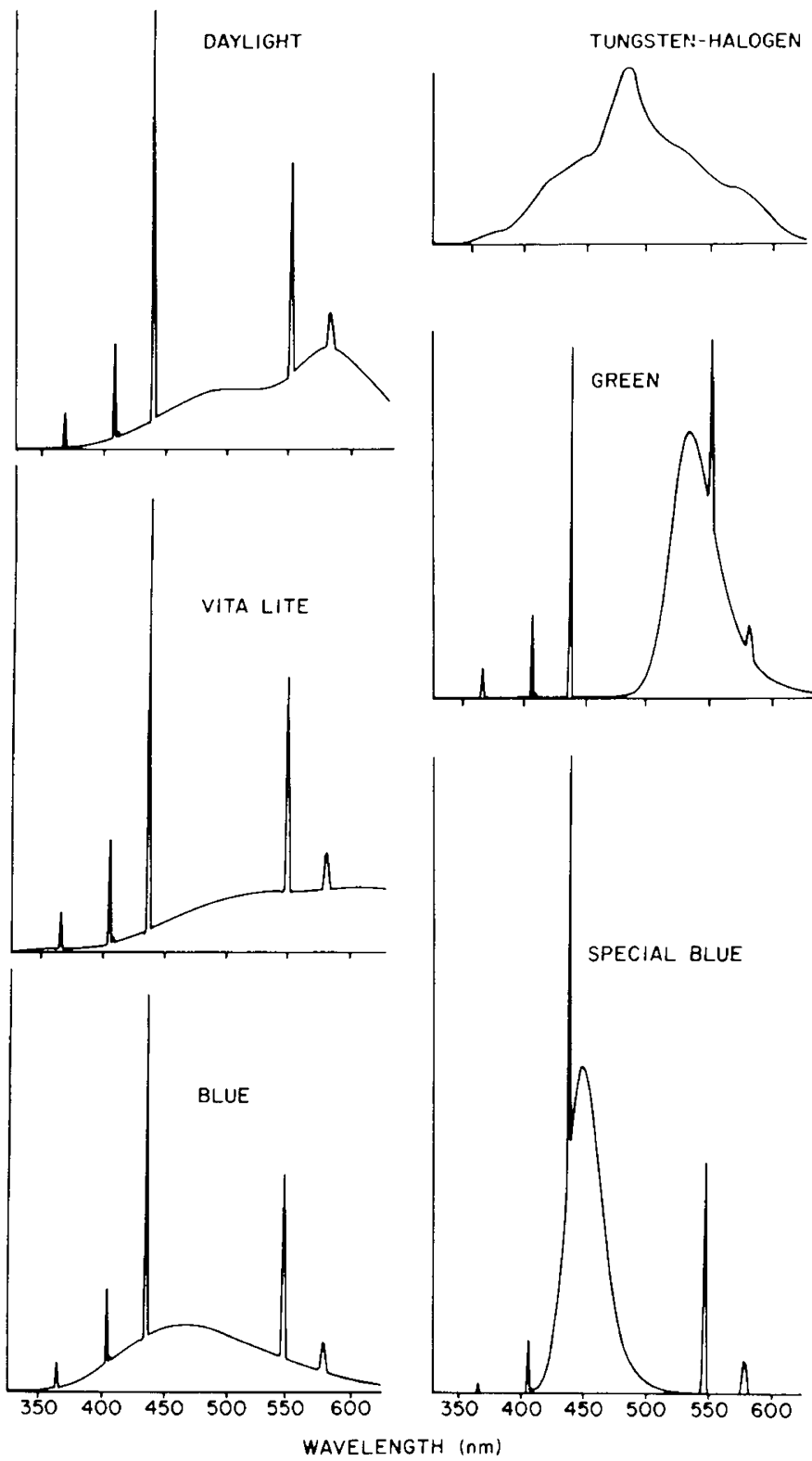

Fig. 1. Emission spectra of phototherapy lamps. The intensities are shown on a linear relative scale and were measured as described in "Materials and Methods." The spectra of the five fluorescent lamps (daylight. Vita-Lite. blue. special blue, and green) were measured under identical conditions on a Cary 118 spectrophotometer. The spectrum of the tungsten-halogen lamp was measured on a Cary 210 spectrophotometer.
This study provides the first comparison of the efficacy of clinically used phototherapy light sources in the biologically important configurational and structural isomerization reactions of bilirubin in vitro. These data cannot be extrapolated directly to in vivo phototherapy because of the influence of the optical properties of the skin (1), and because the relative contributions of configurational and structural isomerization to the therapeutic response to phototherapy are not known. The absorption of light by the skin will diminish the effective irradiance in vivo and thus diminish the initial rates of these reactions. In addition, light scattering preferentially attenuates the shorter wavelengths (1) and thereby alters the effective spectral distribution in vivo and the relative rates of lumirubin and $4 Z, 15 E$ isomer formation. In particular, light sources such as the daylight lamp whose emission within the bilirubin absorption band contains a substantial contribution from shorter wavelengths would probably be less effective in vivo than suggested by our in vitro results.

Certain conclusions can be drawn about the in vivo efficacy of commonly used phototherapy lamps based upon our in vitro data. The broad spectrum fluorescent lamps (daylight, Vita-Lite, and blue) and the green lamp produce the lowest rates of configurational and structural isomerization, and therefore are probably least effective in vivo. The special blue and the tungstenhalogen lamps produce rapid rates of isomerization and are therefore probably the most effective of lamps currently used clinically.

A recently completed clinical study suggests that lumirubin formation and excretion may be an important pathway for bilirubin elimination during phototherapy (Ennever et al., submitted). If lumirubin formation is important and if it is favored. as our in vitro data suggest, by longer wavelength light, then a more effective lamp for phototherapy would be one with a spectral output similar to that of the special blue with its emission band shifted or broadened on the longer wavelength side.

\section{REFERENCES AND NOTES}

1. Anderson RR. Parrish JA 1981 The optics of human skin. J Invest Dermato $77: 13$

2. Ballowitz L, Geutler G, Krochmann J, Pannitschka R, Roemer G, Roemer 1977 Phototherapy in Gunn rats-a study to assess the photobiologically most effective radiant energy and dose/response relationships. Biol Neonate $31: 229$

3. Brown AK. McDonagh AF 1980 Phototherapy for neonatal hyperbilirubine. mia: efficiency. mechanism and toxicity. Adv Pediatr 27:341

4. Ennever JF, Speck WT 1984 Mechanism of action of phototherapy: new concepts. In: Rubaltelli FF. Jori G (eds): New Trends in Phototherapy. New York. Plenum Press, pp 187-196

5. Deleted in proof

6. Ennever JF, McDonagh AF. Speck WT 1983 Phototherapy for neonatal jaundice: optimal wavelengths of light. J Pediatr 103:295

7. Jagger J 1977 Phototechnology and biological experimentation. In: Smith KC (ed): The Science of Photobiology. New York, Plenum Press, pp 1-26

8. Lamola AA. Blumberg WE. McClead R. Fanaroff A 1981 Photoisomerized bilirubin in blood from infants receiving phototherapy. Proc Natl Acad Sci USA 78:1882

Table 1. Isomerization of bilimbin by different phototherapy light sources

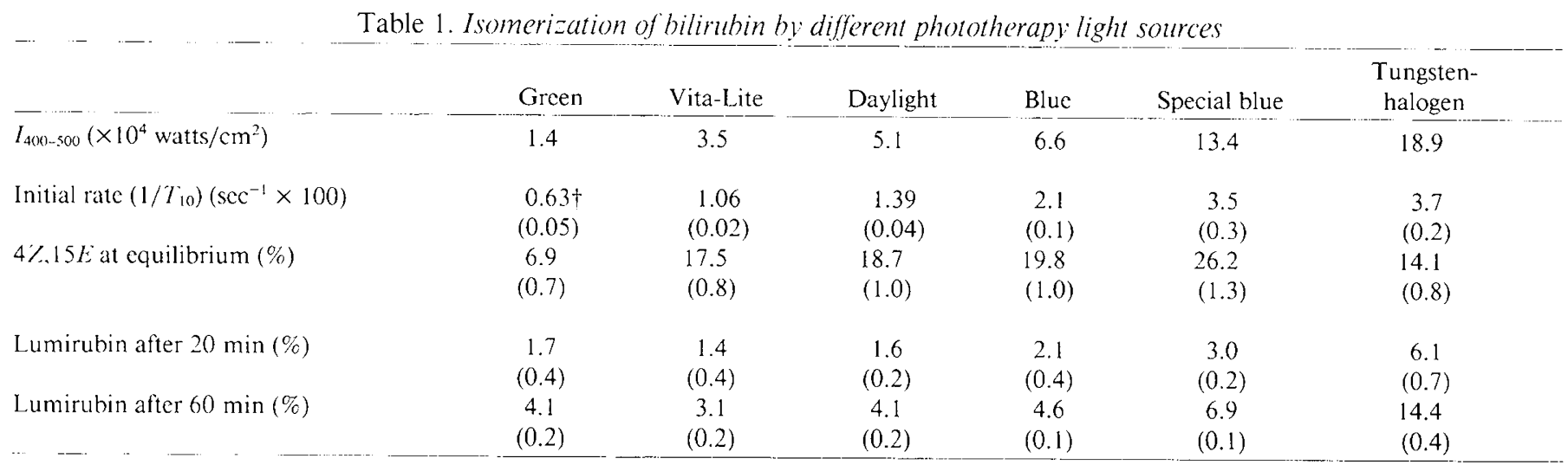

* Values in parentheses are the standard deviation of three or more experiments

+ Calculated by extrapolation from initial rate of configurational isomerization becausc final percentage of $4 Z, 15 E$ isomer was only 6.9 . 
9. McDonagh AF, Assisi F 1972 The ready isomerization of bilirubin $2 \mathrm{X}_{\alpha}$ in aqueous solution. Biochem J 129:797

10. McDonagh AF, Palma LA 1980 Hepatic excretion of circulating bilirubin photoproducts in the Gunn rat. J Clin Invest 66:1182

11. McDonagh AF, Palma LA, Lightner DA 1982 Phototherapy for neonatal jaundice. Stereospecific and regioselective photoisomerization of bilirubin bound to human serum albumin and NMR characterization of intramolecularly cyclized photoproducts. J Am Chem Soc 104:6867

12. McDonagh AF, Palma LA, Trull FR, Lightner DA 1982 Phototherapy for neonatal jaundice. Configurational isomers of bilirubin. J Am Chem Soc 104:6865

13. Onishi S, Isobe K, Itoh S, Kawade N, Sugiyama S 1980 Demonstration of a geometric isomer of bilirubin IX $\alpha$ in the serum of a hyperbilinubinemic newborn infant and the mechanism of jaundice phototherapy. Biochem $\mathrm{J}$ 190:533

14. Onishi S, Kawade N, Itoh S, Isobe K, Sugiyama S, Hashimoto T, Narita H 1981 Kinetics of biliary excretion of the main two bilirubin photoproducts after injection into Gunn rats. Biochem J 198:107

15. Sisson TCR, Kendall N, Shaw A, Kechavarz-Oliai L 1972 Phototherapy of neonatal jaundice in the newborn. II. Effect of various light intensities. J Pediatr 81:35

16. Tan KL 1977 The nature of the dose-response relationship of phototherapy for neonatal hyperbilirubinemia. J Pediatr 90:448

17. Tan KL 1982 The pattern of bilirubin response to phototherapy for neonatal hyperbilirubinemia. Pediatr Res 16:670

18. Vecchi C, Donzelli GP, Migliorini MG, Sbrana G 1983 Green light in phototherapy. Pediatr Res 17:461

19. Warshaw JB, Gagliardi RN, Patel A 1980 A comparison of fluorescent and nonfluorescent light sources for phototherapy. Pediatrics 65:795

20. Requests for reprints should be addressed to: John F. Ennever, Ph.D., M.D., Department of Pediatrics, Rainbow Babies and Childrens Hospital, 2101 Adelbert Road, Cleveland, $\mathrm{OH} 44106$.

21. This research was supported by the United States Public Health Service through Grants CA-23692, AM-26307, and AM-11275, by Research Career Development Award IK0-0043 to W. T. S., and by a grant from the Board of Trustees of Rainbow Babies and Childrens Hospital

22. Received for publication September 26, 1983.

\title{
A New Form of Insulin Resistance with Growth Retardation, Fatty Liver, and Hypogonadotropic Hypogonadism
}

\author{
ETSURO TOKUHIRO, HEATHER DEAN, JEREMY WINTER, JAMES C. HAWORTH, \\ YASUO IMAI, AND HENRY G. FRIESEN ${ }^{(39)}$ \\ Departments of Physiology [E.T., Y.I., H.G.F.] and Pediatrics [H.D., J.W., J.C.H.], Faculty of Medicine, \\ University of Manitoba, Winnipeg, Manitoba, Canada R3E OW3
}

\section{Summary}

A 17-year-old boy presented with growth retardation, marked hepatomegaly, and sexual infantilism. Elevated fasting serum insulin levels and a blunted hypoglycemic response to exogenous insulin (up to 0.35 unit $/ \mathrm{kg}$ ) demonstrated severe insulin resistance. Neither anti-insulin nor anti-insulin receptor antibodies were present. The molecular size of his circulating insulin and its binding to IM-9 lymphocytes was normal. Despite high circulating insulin values, both erythrocytes and cultured skin fibroblasts showed normal insulin binding capacity and affinity. Tissue responsiveness was examined by measuring the insulin-induced increase in 2-deoxyglucose uptake into fibroblasts. Although the basal glucose transport rate was slightly lower than that of controls, the insulin-induced increase was normal. However, the normal increase in thymidine incorporation in response to insulin was blunted, as were the thymidine incorporation responses to epidermal growth factor and fibroblast growth factor. These studies demonstrate the possible existence of a new form of postinsulin receptor defect as a cause of insulin resistance, but underscore the difficulty that exists in defining the exact nature of the defect in these disorders.

IRI, immunoreactive insulin

\section{Abbreviations}

G, gonads

$\mathrm{PH}$, pubic hair

EGF, epidermal growth factor

hFGF, human fibroblast growth factor

Hepes, 4-(2-hydroxyethyl)-1-piperazineethanesulfonic acid 2-DG, 2-deoxyglucose
Insulin resistance, a clinical syndrome characterized by a blunted response to endogenous insulin, with or without a similar poor response to administered insulin, causes some degree of hyperglycemia as well as other more variable clinical features (2, $26)$. Its etiology may include production of an abnormal insulin molecule $(6,33)$, the presence of circulating antagonists to insulin such as anti-insulin or anti-insulin receptor antibodies and contra-insulin factors $(5,25,36)$, abnormally rapid degradation of insulin (27), quantitative (32) and qualitative $(18,35)$ abnormalities of the insulin receptors (18), and poorly defined defects in post-receptor mechanisms $(19,22)$.

This report describes a 17 -year-old boy with an unusual form of insulin resistance in whom extensive studies point to a postreceptor defect but have failed to define the exact nature of the abnormality.

\section{CASE REPORT}

A 17-year-old Caucasian boy was referred for evaluation of hepatomegaly and growth retardation. He was the product of an uneventful 37 -week pregnancy with a birth weight of $2.2 \mathrm{~kg}$. He showed no dysmorphic features other than bilateral cryptorchidism. There was no family history of consanguinity or diabetes. The midparental stature was $161.3 \mathrm{~cm}$. His height and weight growth was below but parallel to the third percentile throughout childhood. Orchidopexy was carried out at 6 years of age; at age 8 , his serum follicle-stimulating hormone, luteinizing hormone, and testosterone values were in the normal prepubertal range. At age 15, hepatomegaly was first noticed. Biopsy of the liver demonstrated severe fatty metamorphosis; there was appreciable glycogen content and moderate periportal fibrosis with some bile 